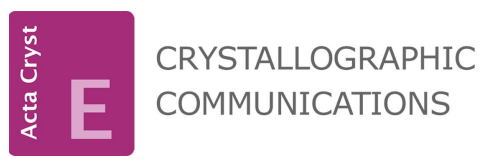

ISSN 2056-9890

Received 25 June 2015

Accepted 11 September 2015

Edited by M. Zeller, Youngstown State University, USA

Keywords: crystal structure; aluminium; indolyl; $\mathrm{C}-\mathrm{H} \cdots \pi$ interactions

CCDC reference: 1423793

Supporting information: this article has supporting information at journals.iucr.org/e

\section{Synthesis, characterization and crystal structure of a 2-(diethylaminomethyl)indole ligated dimethyl- aluminium complex}

\author{
Logan E. Shephard and Nicholas B. Kingsley*
}

Department of Chemistry and Biochemistry, 556 MSB, 303 E. Kearsley, Flint, MI 48502, USA. *Correspondence e-mail:
kingsley@umflint.edu

The title compound, $\left[\mathrm{Al}\left(\mathrm{CH}_{3}\right)_{2}\left(\mathrm{C}_{13} \mathrm{H}_{17} \mathrm{~N}_{2}\right)\right]$ (systematic name; $\{2-[($ diethylamino)methyl]indol-1-yl- $\left.\kappa^{2} N, N^{\prime}\right\}$ dimethylaluminium), was prepared by methane elimination from the reaction of 2-(diethylaminomethyl)indole and trimethylaluminium. The complex crystallizes readily from a concentrated toluene solution in high yield. The asymmetric unit contains two crystallographically independent molecules. Each molecule has a four-coordinate aluminium atom that has pseudo-tetrahedral geometry. $\mathrm{C}-\mathrm{H} \cdots \pi$ interactions link the independent molecules into chains extending along the $b$-axis direction.

\section{Chemical context}

Organoaluminium chemistry has a long history of active research that has led to numerous applications in industry (Mason, 2005). Organoaluminium compounds have garnered much attention in recent years for their use in the formation of polyactides, (Liu et al., 2010; Chisholm et al., 2003, 2005; Zhang et al., 2014; Chen et al., 2012; Schwarz et al., 2010) and hydroamination (Koller \& Bergman, 2010a,b; Khandelwal \& Wehmschulte, 2012). While many varieties of ancillary ligands on aluminium have been employed in such reactions, a majority of these systems have nitrogen-donor arms as a component. Our group is interested in particular in the use of 2-(dialkylaminomethyl)indoles (Nagarathnam, 1992) as ligands for organoaluminium complexes. Herein we report the synthesis, characterization and crystal structure of the first 2-(dialkylaminomethyl)indolyl-aluminium complex, $\left[\mathrm{Al}\left(\mathrm{CH}_{3}\right)_{2}\left(\mathrm{C}_{13} \mathrm{H}_{17} \mathrm{~N}_{2}\right)\right]$.

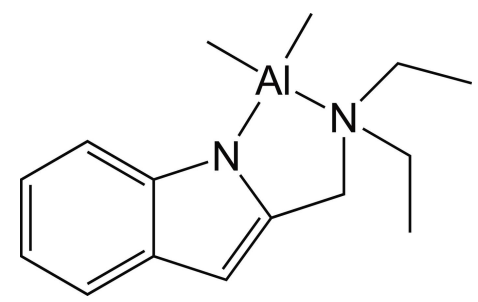

\section{Structural commentary}

The asymmetric unit of the title complex contains two independent molecules (Fig. 1). They are structurally different with regard to the chelate rings that are formed around the aluminium atoms by the indolyl moiety. The most obvious difference between the two crystallographically independent molecules is the displacement of the $\mathrm{Al}$ atom from the plane of the chelate ring. Al1 deviates by 0.6831 (5) $\AA$ from the plane 


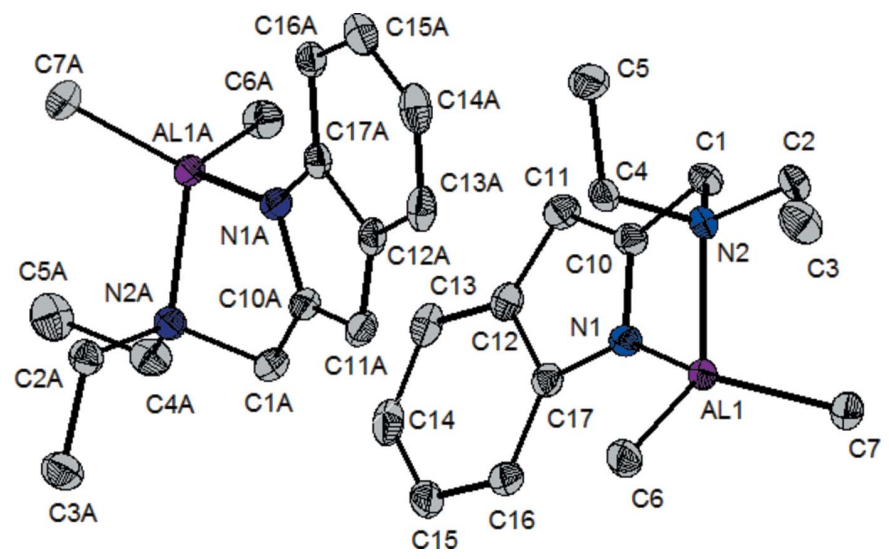

Figure 1

A view of the asymmetric unit of the title compound, showing the atom labeling. Displacement ellipsoids are drawn at the $50 \%$ probability level. $\mathrm{H}$ atoms have been omitted for clarity.

defined by atoms $\mathrm{N} 1 / \mathrm{C} 10 / \mathrm{C} 1 / \mathrm{N} 2$ while Al1 $A$ deviates by 0.6150 (5) $\AA$ from the plane $\mathrm{N} 1 A / \mathrm{C} 10 A / \mathrm{C} 1 A / \mathrm{N} 2 A$. Each molecule contains a four-coordinate, pseudo-tetrahedral, aluminium atom. There are two distinct bond lengths for the $\mathrm{Al}-\mathrm{N}$ bonds in the molecule. The $\mathrm{Al}-\mathrm{N}_{\text {indolyl }}$ bond lengths are 1.8879 (14) $\AA$ for Al1 - N1 and 1.8779 (15) $\AA$ for Al1 $A-$ $\mathrm{N} 1 A$. These lengths are in the range expected for anionically bound indolyl or pyrrolyl moieties (Huang et al., 2001). As expected, these lengths are significantly shorter than those found for the dative $\mathrm{Al}-\mathrm{N}_{\text {imine }}$ bonds, 2.0355 (15) $\AA$ for $\mathrm{Al} 1-$ $\mathrm{N} 2$ and 2.0397 (16) $\AA$ for Al1 $A-\mathrm{N} 2 A$ [see Huang et al. (2001) for typical values].

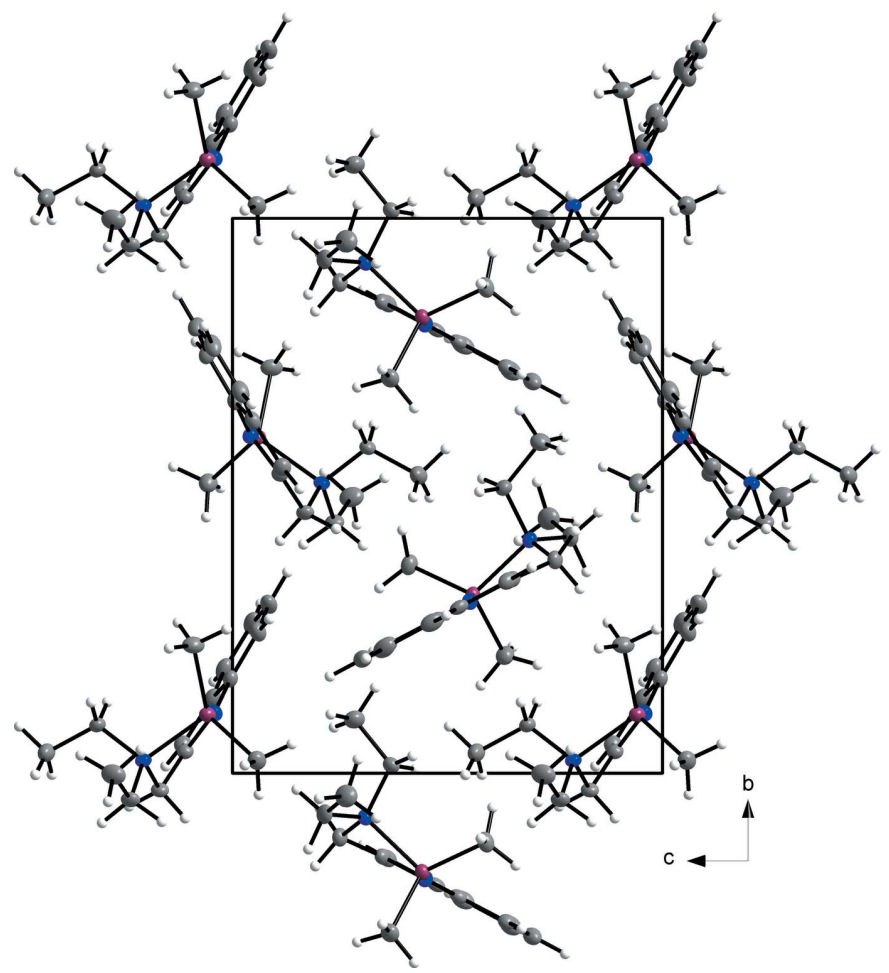

Figure 2

Crystal packing diagram of the title compound viewed along the $a$ axis.

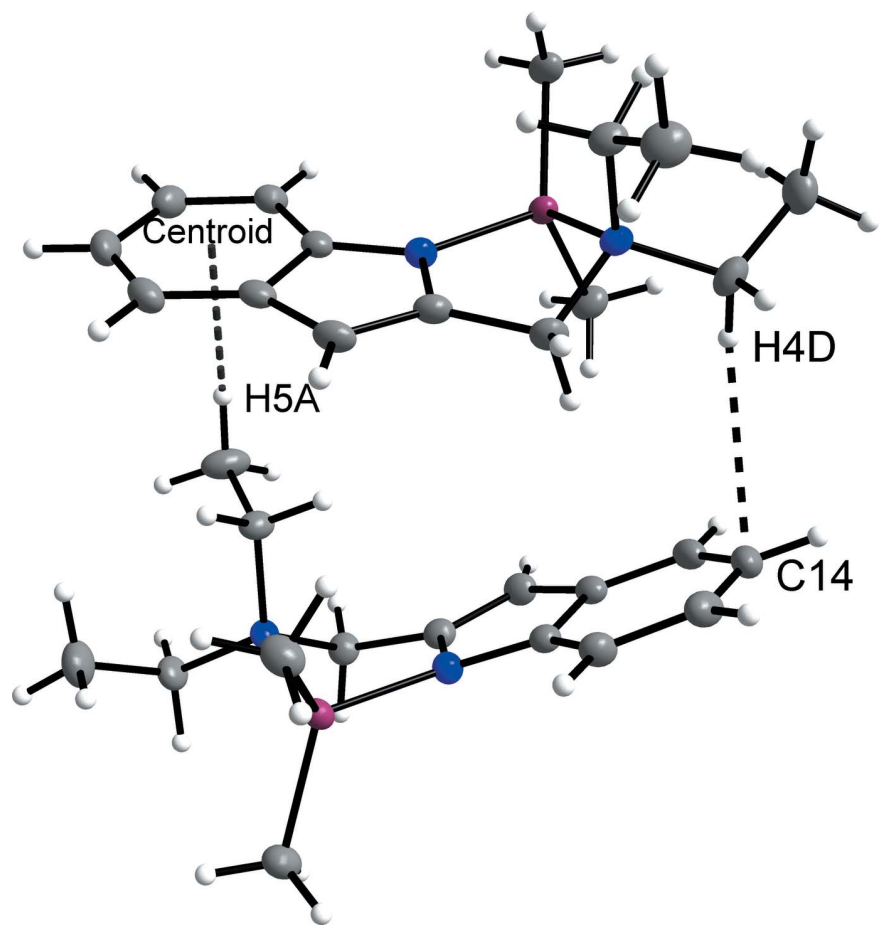

Figure 3

$\mathrm{C}-\mathrm{H} \cdots \pi$ interactions between molecules in the asymmetric unit.

\section{Supramolecular features}

The crystal packing is illustrated in Fig. 2. In the crystal, molecules associate via three different types of $\mathrm{C}-\mathrm{H} \cdots \pi$ interactions, as shown in Figs. 3 and 4. There is one interaction between the methyl proton $\mathrm{H} 5 A$ and the centroid of the $(\mathrm{C} 12 A-\mathrm{C} 17 A)$ aromatic ring of $2.57 \AA$ (Table 1$)$ and another between the methylene proton $\mathrm{H} 4 D$ and the aromatic $\mathrm{C} 14$ of $2.88 \AA$. The third interaction is between $\mathrm{H} 2 B$ and the centroid of $\mathrm{C} 12 A^{\mathrm{i}}-\mathrm{C} 17 A^{\mathrm{i}}$ [Table 1 ; symmetry code: (i) $1-x,-\frac{1}{2}+y$, $1-z]$. This interaction links the two independent molecules in the asymmetric unit into chains that extend along the $b$-axis direction.

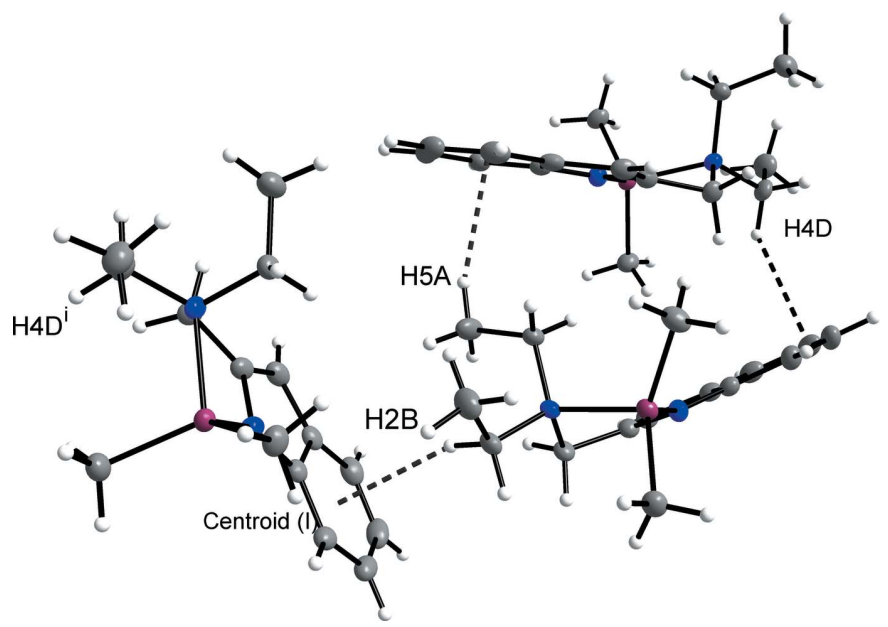

Figure 4

All $\mathrm{C}-\mathrm{H} \cdots \pi$ interactions between molecules of the title compound. [Symmetry code: (i) $1-x,-\frac{1}{2}+y, 1-z$.] 
Table 1

$\mathrm{C}-\mathrm{H} \cdots \pi$ interactions $\left(\AA,{ }^{\circ}\right)$.

$C g 1$ is the centroid of the $\mathrm{C} 12 A-\mathrm{C} 17 A$ ring.

\begin{tabular}{lllll}
\hline$D-\mathrm{H} \cdots A$ & $D-\mathrm{H}$ & $\mathrm{H} \cdots A$ & $D \cdots A$ & $D-\mathrm{H} \cdots A$ \\
\hline $\mathrm{C} 5-\mathrm{H} 5 A \cdots C g 1$ & 0.98 & 2.57 & $3.470(2)$ & 153 \\
$\mathrm{C} 2-\mathrm{H} 2 B \cdots C g 1^{\mathrm{i}}$ & 0.99 & 2.55 & $3.434(2)$ & 149
\end{tabular}

Symmetry code: (i) $-x+1, y-\frac{1}{2},-z+1$.

\section{Database survey}

A search of the Cambridge Structural Database (CSD, Version 5.36; Groom \& Allen, 2014) for indolyl gave 500 hits. A search for indolide generated 18 hits. Neither of these sets of hits included structures involving indolyl moieties bound to aluminium. A substructure search for N-bound indolylcoordinating aluminium complexes resulted in only five hits (Kingsley et al., 2010), all of which contained bridging $\mu^{2}: \eta^{1}: \eta^{1}$ coordination modes. The title compound is the first structurally characterized complex with a monomeric $\mu^{1}: \eta^{1}$ coordinating indole moiety to aluminium.

\section{Synthesis and crystallization}

To a $100 \mathrm{~mL}$ side-arm flask was added 2-(diethylaminomethyl)indole $(0.402 \mathrm{~g}, 2.0 \mathrm{mmol})$ and $25 \mathrm{~mL}$ of toluene. A toluene solution of trimethylaluminium $(1.0 \mathrm{~mL}, 2.0 \mathrm{M}, 2.0 \mathrm{mmol})$ was added via syringe. The reaction solution turned bright yellow, which darkened as the solution was stirred for $12 \mathrm{~h}$. The solvent was then removed in vacuo resulting in a yellow solid, which was dissolved in a mixture of $10 \mathrm{~mL}$ of hot toluene, followed by cooling to $243 \mathrm{~K}$ for $48 \mathrm{~h}$. The resulting yellow crystalline material was isolated by filtration. Yield: $0.462 \mathrm{~g}$, $1.78 \mathrm{mmol}, 90 \% .{ }^{1} \mathrm{H}$ NMR $\left(\mathrm{CDCl}_{3}, 600 \mathrm{MHz}\right): \delta 7.55\left(d,{ }^{3} J_{\mathrm{HH}}\right.$ $=7.8 \mathrm{~Hz}, 1 \mathrm{H}, \mathrm{H} 16), 7.36\left(d,{ }^{3} J_{\mathrm{HH}}=7.8 \mathrm{~Hz}, 1 \mathrm{H}, \mathrm{H} 13\right), 7.07(t$, $\left.{ }^{3} J_{\mathrm{HH}}=7.8 \mathrm{~Hz}, 1 \mathrm{H}, \mathrm{H} 15\right), 7.00\left(t,{ }^{3} J_{\mathrm{HH}}=7.8 \mathrm{~Hz}, 1 \mathrm{H}, \mathrm{H} 14\right), 6.31$ $(s, 1 \mathrm{H}, \mathrm{H} 11), 4.00\left(s, 2 \mathrm{H}\right.$, indole $\left.\mathrm{CH}_{2}\right), 2.88\left(q,{ }^{3} J_{\mathrm{HH}}=7.2 \mathrm{~Hz}\right.$, $4 \mathrm{H}$, amino $\left.\mathrm{CH}_{2} \mathrm{CH}_{3}\right), 1.13\left(t,{ }^{3} \mathrm{~J}_{\mathrm{HH}}=7.2 \mathrm{~Hz}, 6 \mathrm{H}\right.$, amino $\left.\left.\mathrm{CH}_{2} \mathrm{CH}_{3}\right),-0.59\left(s, 6 \mathrm{H}, \mathrm{AlCH}_{3}\right) \cdot{ }^{13} \mathrm{C}_{1}{ }^{1} \mathrm{H}\right\}$ NMR $\left(\mathrm{CDCl}_{3}\right.$, $150.8 \mathrm{MHz}$ ): $\delta 141.7$ (C17), 139.4 (C10), 131.8 (C12), 120.2 (C15), 119.6 (C16), 118.5 (C15), 113.7 (C14), 98.1 (C11), 53.2 (indole $\mathrm{CH}_{2}$ ), 44.7 (amino $\mathrm{CH}_{2} \mathrm{CH}_{3}$ ), $8.3\left(\right.$ amino $\mathrm{CH}_{2} \mathrm{CH}_{3}$ ), $-11.10\left(\mathrm{br}, \mathrm{AlCH}_{3}\right)(\mathrm{Kingsley}$ et al., 2010). Analysis calculated for $\mathrm{C}_{15} \mathrm{H}_{23} \mathrm{~N}_{2} \mathrm{Al}$ : C, 69.74; H, 8.97; N, 10.84. Found: C, 69.67; H, $8.70 ; \mathrm{N}, 10.63$.

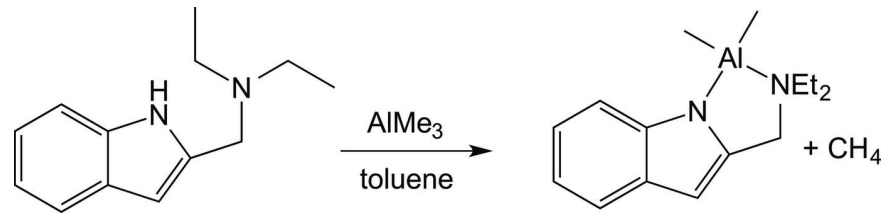

X-ray quality crystals were grown from a concentrated solution in hot toluene followed by slow cooling to room temperature followed by storage at $243 \mathrm{~K}$ for $72 \mathrm{~h}$.
Table 2

Experimental details.

\begin{tabular}{|c|c|}
\hline \multicolumn{2}{|l|}{ Crystal data } \\
\hline Chemical formula & {$\left[\mathrm{Al}\left(\mathrm{CH}_{3}\right)_{2}\left(\mathrm{C}_{13} \mathrm{H}_{17} \mathrm{~N}_{2}\right)\right]$} \\
\hline$M_{\mathrm{r}}$ & 258.33 \\
\hline Crystal system, space group & Monoclinic, $P 2_{1}$ \\
\hline Temperature $(\mathrm{K})$ & 150 \\
\hline$a, b, c(\AA)$ & $9.7467(5), 14.1245(7), 10.9866(5)$ \\
\hline$\beta\left(^{\circ}\right)$ & $94.206(1)$ \\
\hline$V\left(\AA^{3}\right)$ & $1508.42(13)$ \\
\hline$Z$ & 4 \\
\hline Radiation type & Mo $K \alpha$ \\
\hline$\mu\left(\mathrm{mm}^{-1}\right)$ & 0.12 \\
\hline Crystal size $(\mathrm{mm})$ & $0.20 \times 0.20 \times 0.15$ \\
\hline \multicolumn{2}{|l|}{ Data collection } \\
\hline Diffractometer & Bruker APEXII CCD \\
\hline Absorption correction & $\begin{array}{l}\text { Multi-scan ( } S A D A B S ; \text { Bruker, } \\
\text { 2003) }\end{array}$ \\
\hline$T_{\min }, T_{\max }$ & $0.697,0.745$ \\
\hline $\begin{array}{l}\text { No. of measured, independent and } \\
\text { observed }[I>2 \sigma(I)] \text { reflections }\end{array}$ & $13157,5440,5366$ \\
\hline$R_{\text {int }}$ & 0.025 \\
\hline$(\sin \theta / \lambda)_{\max }\left(\AA^{-1}\right)$ & 0.624 \\
\hline \multicolumn{2}{|l|}{ Refinement } \\
\hline$R\left[F^{2}>2 \sigma\left(F^{2}\right)\right], w R\left(F^{2}\right), S$ & $0.024,0.068,1.05$ \\
\hline No. of reflections & 5440 \\
\hline No. of parameters & 333 \\
\hline No. of restraints & 1 \\
\hline $\mathrm{H}$-atom treatment & H-atom parameters constrained \\
\hline$\Delta \rho_{\max }, \Delta \rho_{\min }\left(\mathrm{e} \AA^{-3}\right)$ & $0.21,-0.19$ \\
\hline Absolute structure & $\begin{array}{l}\text { Flack } x \text { determined using } 2203 \\
\quad \text { quotients }\left[\left(I^{+}\right)-\left(I^{-}\right)\right] /\left[\left(I^{+}\right)+\left(I^{-}\right)\right] \\
\quad \text { (Parsons } \text { et al. }, 2013)\end{array}$ \\
\hline Absolute structure parameter & $0.05(3)$ \\
\hline
\end{tabular}

Computer programs: APEX2 (Bruker, 2005), SAINT (Bruker, 2003), SHELXS97 (Sheldrick, 2008), SHELXL2014 (Sheldrick, 2015), DIAMOND (Brandenburg, 2010) and publCIF (Westrip, 2010).

\section{Refinement}

Crystal data, data collection and structure refinement details are summarized in Table 2. All $\mathrm{H}$ atoms were positioned geometrically and refined using a riding model with $\mathrm{C}-\mathrm{H}=$ $0.05-0.99 \AA$ and $U_{\text {iso }}(\mathrm{H})=1.2$ or $1.5 U_{\text {eq }}(\mathrm{C})$.

\section{Acknowledgements}

The authors would like to thank the University of MichiganFlint Office of Research and Sponsored Programs for their support of this project. Special acknowledgement is given to Dr Chris Gianopoulos for assistance in data collection and structure refinement and to the University of Toledo Instrumentation Center for the use of their Bruker APEXII diffractometer.

\section{References}

Brandenburg, K. (2010). DIAMOND. Crystal Impact GbR, Bonn, Germany.

Bruker (2003). SADABS and SAINT. Bruker AXS Inc., Madison, Wisconsin, USA.

Bruker (2005). APEX2. Bruker AXS Inc., Madison, Wisconsin, USA. Chen, H.-L., Dutta, S., Huang, P.-Y. \& Lin, C.-C. (2012). Organometallics, 31, 2016-2025. 
Chisholm, M. H., Lin, C.-C., Gallucci, J. C. \& Ko, B. T. (2003). Dalton Trans. pp. 406-412.

Chisholm, M. H., Patmore, N. J. \& Zhou, Z. (2005). Chem. Commun. pp. 127-129.

Groom, C. R. \& Allen, F. H. (2014). Angew. Chem. Int. Ed. 53, 662671.

Huang, J., Chen, H., Chang, C., Zhou, C., Lee, G. \& Peng, S. (2001). Organometallics, 20, 2647-2650.

Khandelwal, M. \& Wehmschulte, R. J. (2012). J. Organomet. Chem. 696, 4179-4183.

Kingsley, N. B., Kirschbaum, K. \& Mason, M. R. (2010). Organometallics, 29, 5927-5935.

Koller, J. \& Bergman, R. G. (2010a). Chem. Commun. 46, 4577-4579.

Koller, J. \& Bergman, R. G. (2010b). Organometallics, 29, 5946-5952.
Liu, Z., Gao, W., Zhang, J., Cui, D., Wu, Q. \& Mu, Y. (2010). Organometallics, 29, 5783-5790.

Mason, M. R. (2005). Encyclopedia of Inorganic Chemistry, Vol. 1, 2nd ed., edited by B. King, pp. 185-210. Hoboken, NJ: Wiley.

Nagarathnam, D. (1992). Synthesis, pp. 743-745.

Parsons, S., Flack, H. D. \& Wagner, T. (2013). Acta Cryst. B69, 249 259.

Schwarz, A. D., Chu, Z. \& Mountford, P. (2010). Organometallics, 29, 1246-1260.

Sheldrick, G. M. (2008). Acta Cryst. A64, 112-122.

Sheldrick, G. M. (2015). Acta Cryst. C71, 3-8.

Westrip, S. P. (2010). J. Appl. Cryst. 43, 920-925.

Zhang, W., Wang, Y., Wang, L., Redshaw, C. \& Sun, W.-H. (2014). J. Organomet. Chem. 750, 65-73. 


\section{supporting information}

Acta Cryst. (2015). E71, 1222-1225 [doi:10.1107/S2056989015017053]

\section{Synthesis, characterization and crystal structure of a 2-(diethylaminomethyl)- indole ligated dimethylaluminium complex}

\section{Logan E. Shephard and Nicholas B. Kingsley}

\section{Computing details}

Data collection: APEX2 (Bruker, 2005); cell refinement: SAINT (Bruker, 2003); data reduction: SAINT (Bruker, 2003); program(s) used to solve structure: SHELXS97 (Sheldrick, 2008); program(s) used to refine structure: SHELXL2014 (Sheldrick, 2015); molecular graphics: DIAMOND (Brandenburg, 2010); software used to prepare material for publication: publCIF (Westrip, 2010)..

\section{$\left\{2-\left[\left(\right.\right.\right.$ Diethylamino)methyl]indol-1-yl- $\left.\kappa^{2} N, N^{\prime}\right\}$ dimethylaluminium}

Crystal data

$$
\begin{aligned}
& {\left[\mathrm{Al}\left(\mathrm{CH}_{3}\right)_{2}\left(\mathrm{C}_{13} \mathrm{H}_{17} \mathrm{~N}_{2}\right)\right]} \\
& M_{r}=258.33 \\
& \text { Monoclinic, } P 2_{1} \\
& a=9.7467(5) \AA \\
& b=14.1245(7) \AA \\
& c=10.9866(5) \AA \\
& \beta=94.206(1)^{\circ} \\
& V=1508.42(13) \AA^{3} \\
& Z=4
\end{aligned}
$$

\section{Data collection}

Bruker APEXII CCD diffractometer

Radiation source: sealed tube $\varphi$ and $\omega$ scans

Absorption correction: multi-scan (SADABS; Bruker, 2003)

$T_{\min }=0.697, T_{\max }=0.745$

13157 measured reflections

\section{Refinement}

Refinement on $F^{2}$

Least-squares matrix: full

$R\left[F^{2}>2 \sigma\left(F^{2}\right)\right]=0.024$

$w R\left(F^{2}\right)=0.068$

$S=1.05$

5440 reflections

333 parameters

1 restraint

Hydrogen site location: inferred from neighbouring sites

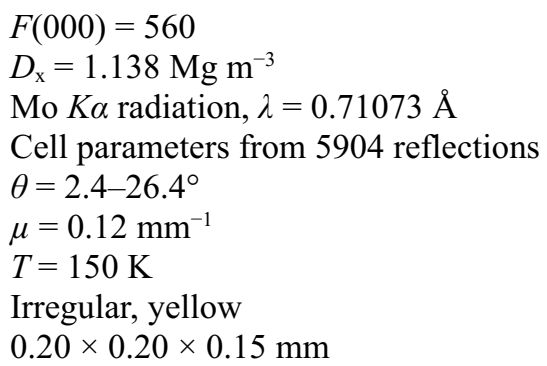

5440 independent reflections 5366 reflections with $I>2 \sigma(I)$

$R_{\text {int }}=0.025$

$\theta_{\text {max }}=26.3^{\circ}, \theta_{\text {min }}=1.9^{\circ}$

$h=-12 \rightarrow 10$

$k=-16 \rightarrow 17$

$l=-13 \rightarrow 12$

H-atom parameters constrained

$w=1 /\left[\sigma^{2}\left(F_{\mathrm{o}}^{2}\right)+(0.0388 P)^{2}+0.2513 P\right]$

where $P=\left(F_{\mathrm{o}}^{2}+2 F_{\mathrm{c}}^{2}\right) / 3$

$(\Delta / \sigma)_{\max }<0.001$

$\Delta \rho_{\max }=0.21 \mathrm{e} \AA^{-3}$

$\Delta \rho_{\text {min }}=-0.19$ e $\AA^{-3}$

Absolute structure: Flack $x$ determined using 2203 quotients $\left[\left(I^{+}\right)-\left(I^{-}\right)\right] /\left[\left(I^{+}\right)+\left(I^{-}\right)\right]$(Parsons et al., 2013)

Absolute structure parameter: 0.05 (3) 


\section{Special details}

Geometry. All e.s.d.'s (except the e.s.d. in the dihedral angle between two 1.s. planes) are estimated using the full covariance matrix. The cell e.s.d.'s are taken into account individually in the estimation of e.s.d.'s in distances, angles and torsion angles; correlations between e.s.d.'s in cell parameters are only used when they are defined by crystal symmetry. An approximate (isotropic) treatment of cell e.s.d.'s is used for estimating e.s.d.'s involving l.s. planes.

Fractional atomic coordinates and isotropic or equivalent isotropic displacement parameters $\left(\hat{A}^{2}\right)$

\begin{tabular}{|c|c|c|c|c|}
\hline & $x$ & $y$ & $z$ & $U_{\text {iso }} * / U_{\text {eq }}$ \\
\hline Al1 & $0.49235(5)$ & $0.10530(4)$ & $0.05845(4)$ & $0.01712(12)$ \\
\hline Al1A & $0.00034(5)$ & $0.32156(4)$ & $0.44114(4)$ & $0.01652(12)$ \\
\hline N1 & $0.29822(14)$ & $0.10862(11)$ & $0.04241(13)$ & $0.0187(3)$ \\
\hline N1A & $0.19261(14)$ & $0.30931(11)$ & $0.44801(12)$ & $0.0185(3)$ \\
\hline N2 & $0.46863(14)$ & $0.02289(10)$ & $0.20745(13)$ & $0.0166(3)$ \\
\hline N2A & $0.02362(15)$ & $0.42016(11)$ & $0.30859(12)$ & 0.0185 \\
\hline $\mathrm{C} 1$ & $0.33910(17)$ & $-0.03044(13)$ & $0.16893(16)$ & $0.0194(3)$ \\
\hline $\mathrm{H} 1 \mathrm{~A}$ & 0.3588 & -0.0811 & 0.1105 & $0.023 *$ \\
\hline H1B & 0.3005 & -0.0598 & 0.2408 & $0.023^{*}$ \\
\hline $\mathrm{C} 2$ & $0.58090(17)$ & $-0.04771(13)$ & $0.23735(16)$ & $0.0210(4)$ \\
\hline $\mathrm{H} 2 \mathrm{~A}$ & 0.5922 & -0.0876 & 0.1647 & $0.025^{*}$ \\
\hline $\mathrm{H} 2 \mathrm{~B}$ & 0.5532 & -0.0896 & 0.3035 & $0.025^{*}$ \\
\hline $\mathrm{C} 3$ & $0.7178(2)$ & $-0.00237(15)$ & $0.2769(2)$ & $0.0311(4)$ \\
\hline $\mathrm{H} 3 \mathrm{~A}$ & 0.7883 & -0.0516 & 0.2893 & $0.047^{*}$ \\
\hline H3B & 0.7099 & 0.0322 & 0.3534 & $0.047^{*}$ \\
\hline $\mathrm{H} 3 \mathrm{C}$ & 0.7439 & 0.0417 & 0.2136 & $0.047^{*}$ \\
\hline $\mathrm{C} 4$ & $0.44570(18)$ & $0.08681(13)$ & $0.31419(15)$ & $0.0201(3)$ \\
\hline $\mathrm{H} 4 \mathrm{~A}$ & 0.3664 & 0.1285 & 0.2912 & $0.024 *$ \\
\hline H4B & 0.5276 & 0.1278 & 0.3291 & $0.024^{*}$ \\
\hline $\mathrm{C} 5$ & $0.4190(2)$ & $0.03699(15)$ & $0.43275(17)$ & $0.0319(4)$ \\
\hline $\mathrm{H} 5 \mathrm{~A}$ & 0.4053 & 0.0843 & 0.4960 & $0.048^{*}$ \\
\hline H5B & 0.4981 & -0.0030 & 0.4585 & $0.048^{*}$ \\
\hline $\mathrm{H} 5 \mathrm{C}$ & 0.3365 & -0.0025 & 0.4202 & $0.048^{*}$ \\
\hline C6 & $0.57142(19)$ & $0.23092(14)$ & $0.09103(18)$ & $0.0263(4)$ \\
\hline H6A & 0.6534 & 0.2252 & 0.1478 & $0.039^{*}$ \\
\hline H6B & 0.5035 & 0.2713 & 0.1272 & $0.039^{*}$ \\
\hline $\mathrm{H} 6 \mathrm{C}$ & 0.5969 & 0.2592 & 0.0144 & $0.039^{*}$ \\
\hline $\mathrm{C} 7$ & $0.58197(19)$ & $0.02303(15)$ & $-0.05668(16)$ & $0.0254(4)$ \\
\hline H7A & 0.6789 & 0.0143 & -0.0284 & $0.038^{*}$ \\
\hline H7B & 0.5760 & 0.0524 & -0.1377 & $0.038^{*}$ \\
\hline $\mathrm{H} 7 \mathrm{C}$ & 0.5357 & -0.0386 & -0.0613 & $0.038^{*}$ \\
\hline $\mathrm{C} 10$ & $0.23890(17)$ & $0.03923(13)$ & $0.11001(15)$ & $0.0190(3)$ \\
\hline C11 & $0.10054(18)$ & $0.05168(13)$ & $0.11525(16)$ & $0.0215(4)$ \\
\hline H11 & 0.0391 & 0.0126 & 0.1559 & $0.026^{*}$ \\
\hline $\mathrm{C} 12$ & $0.06711(17)$ & $0.13596(14)$ & $0.04673(16)$ & $0.0204(4)$ \\
\hline $\mathrm{C} 13$ & $-0.05440(18)$ & $0.18738(15)$ & $0.01868(16)$ & $0.0256(4)$ \\
\hline H13 & -0.1397 & 0.1651 & 0.0442 & $0.031^{*}$ \\
\hline $\mathrm{C} 14$ & $-0.0484(2)$ & $0.27035(16)$ & $-0.04612(17)$ & $0.0283(4)$ \\
\hline H14 & -0.1304 & 0.3053 & -0.0655 & $0.034 *$ \\
\hline
\end{tabular}




\begin{tabular}{|c|c|c|c|c|}
\hline $\mathrm{C} 15$ & $0.0767(2)$ & $0.30439(15)$ & $-0.08420(16)$ & $0.0281(4)$ \\
\hline H15 & 0.0783 & 0.3624 & -0.1277 & $0.034 *$ \\
\hline $\mathrm{C} 16$ & $0.19806(19)$ & $0.25458(14)$ & $-0.05925(16)$ & $0.0238(4)$ \\
\hline H16 & 0.2827 & 0.2777 & -0.0852 & $0.029^{*}$ \\
\hline $\mathrm{C} 17$ & $0.19268(17)$ & $0.16992(13)$ & $0.00483(15)$ & 0.0188 \\
\hline $\mathrm{C} 1 \mathrm{~A}$ & $0.14884(18)$ & $0.38445(14)$ & $0.24981(15)$ & $0.0219(4)$ \\
\hline H1D & 0.1228 & 0.3324 & 0.1924 & $0.026^{*}$ \\
\hline H1E & 0.1900 & 0.4361 & 0.2037 & $0.026^{*}$ \\
\hline $\mathrm{C} 2 \mathrm{~A}$ & $0.05447(18)$ & $0.51442(13)$ & $0.36912(15)$ & $0.0216(4)$ \\
\hline $\mathrm{H} 2 \mathrm{D}$ & 0.1376 & 0.5072 & 0.4258 & $0.026^{*}$ \\
\hline $\mathrm{H} 2 \mathrm{E}$ & -0.0229 & 0.5309 & 0.4187 & $0.026^{*}$ \\
\hline $\mathrm{C} 3 \mathrm{~A}$ & $0.0777(2)$ & $0.59665(15)$ & $0.28370(18)$ & $0.0299(4)$ \\
\hline H3D & 0.0966 & 0.6543 & 0.3317 & $0.045^{*}$ \\
\hline $\mathrm{H} 3 \mathrm{E}$ & -0.0047 & 0.6061 & 0.2284 & $0.045^{*}$ \\
\hline $\mathrm{H} 3 \mathrm{~F}$ & 0.1563 & 0.5826 & 0.2359 & $0.045^{*}$ \\
\hline $\mathrm{C} 4 \mathrm{~A}$ & $-0.09529(19)$ & $0.42606(15)$ & $0.21401(16)$ & $0.0252(4)$ \\
\hline $\mathrm{H} 4 \mathrm{D}$ & -0.1106 & 0.3629 & 0.1764 & $0.030^{*}$ \\
\hline $\mathrm{H} 4 \mathrm{E}$ & -0.0714 & 0.4704 & 0.1490 & $0.030^{*}$ \\
\hline $\mathrm{C} 5 \mathrm{~A}$ & $-0.2272(2)$ & $0.45879(17)$ & $0.26472(19)$ & $0.0327(5)$ \\
\hline H5D & -0.3030 & 0.4539 & 0.2013 & $0.049^{*}$ \\
\hline $\mathrm{H} 5 \mathrm{E}$ & -0.2173 & 0.5248 & 0.2914 & $0.049^{*}$ \\
\hline $\mathrm{H} 5 \mathrm{~F}$ & -0.2470 & 0.4190 & 0.3343 & $0.049 *$ \\
\hline C6A & $-0.0962(2)$ & $0.21207(14)$ & $0.36621(17)$ & $0.0259(4)$ \\
\hline H6D & -0.1936 & 0.2277 & 0.3485 & $0.039^{*}$ \\
\hline H6E & -0.0880 & 0.1582 & 0.4225 & $0.039^{*}$ \\
\hline $\mathrm{H} 6 \mathrm{~F}$ & -0.0553 & 0.1955 & 0.2902 & $0.039^{*}$ \\
\hline C7A & $-0.06954(18)$ & $0.37556(15)$ & $0.58896(16)$ & $0.0234(4)$ \\
\hline H7D & -0.1698 & 0.3820 & 0.5775 & $0.035^{*}$ \\
\hline $\mathrm{H} 7 \mathrm{E}$ & -0.0281 & 0.4380 & 0.6048 & $0.035^{*}$ \\
\hline $\mathrm{H} 7 \mathrm{~F}$ & -0.0456 & 0.3337 & 0.6585 & $0.035^{*}$ \\
\hline $\mathrm{C} 10 \mathrm{~A}$ & $0.25049(18)$ & $0.34955(13)$ & $0.34895(15)$ & 0.0198 \\
\hline C11A & $0.39089(18)$ & $0.34640(14)$ & $0.36008(16)$ & $0.0223(4)$ \\
\hline H11A & 0.4520 & 0.3707 & 0.3042 & $0.027^{*}$ \\
\hline $\mathrm{C} 12 \mathrm{~A}$ & $0.42739(18)$ & $0.29896(12)$ & $0.47316(16)$ & $0.0202(4)$ \\
\hline $\mathrm{C} 13 \mathrm{~A}$ & $0.55142(18)$ & $0.27146(14)$ & $0.53626(18)$ & $0.0261(4)$ \\
\hline $\mathrm{H} 13 \mathrm{~A}$ & 0.6369 & 0.2843 & 0.5032 & $0.031^{*}$ \\
\hline $\mathrm{C} 14 \mathrm{~A}$ & $0.54780(19)$ & $0.22547(15)$ & $0.64699(18)$ & $0.0284(4)$ \\
\hline H14A & 0.6316 & 0.2067 & 0.6899 & $0.034 *$ \\
\hline $\mathrm{C} 15 \mathrm{~A}$ & $0.4220(2)$ & $0.20598(14)$ & $0.69735(17)$ & $0.0256(4)$ \\
\hline $\mathrm{H} 15 \mathrm{~A}$ & 0.4225 & 0.1744 & 0.7737 & $0.031^{*}$ \\
\hline $\mathrm{C} 16 \mathrm{~A}$ & $0.29816(18)$ & $0.23198(13)$ & $0.63774(16)$ & 0.0205 \\
\hline H16A & 0.2135 & 0.2192 & 0.6723 & $0.025^{*}$ \\
\hline $\mathrm{C} 17 \mathrm{~A}$ & $0.30091(17)$ & $0.27766(13)$ & $0.52507(15)$ & $0.0179(3)$ \\
\hline
\end{tabular}

Atomic displacement parameters $\left(\AA^{2}\right)$

\begin{tabular}{lllllll}
\hline & $U^{11}$ & $U^{22}$ & $U^{33}$ & $U^{12}$ & $U^{13}$ & $U^{23}$ \\
\hline Al1 & $0.0165(2)$ & $0.0158(3)$ & $0.0194(2)$ & $0.00128(19)$ & $0.00356(18)$ & $0.00057(19)$
\end{tabular}


supporting information

\begin{tabular}{|c|c|c|c|c|c|c|}
\hline AllA & $0.0162(2)$ & $0.0184(3)$ & $0.0150(2)$ & -0.00063 (19) & $0.00145(18)$ & $-0.00094(19)$ \\
\hline N1 & $0.0183(6)$ & $0.0191(8)$ & $0.0189(7)$ & $0.0012(6)$ & $0.0020(5)$ & $0.0021(6)$ \\
\hline N1A & $0.0193(7)$ & $0.0195(8)$ & $0.0168(6)$ & $0.0008(6)$ & $0.0031(5)$ & $0.0009(6)$ \\
\hline $\mathrm{N} 2$ & $0.0177(6)$ & $0.0129(7)$ & $0.0192(6)$ & $0.0013(6)$ & $0.0003(5)$ & $-0.0014(6)$ \\
\hline N2A & $0.0219(7)$ & $0.0181(7)$ & $0.0152(6)$ & 0.0007 (6) & $0.0000(5)$ & $-0.0016(5)$ \\
\hline $\mathrm{C} 1$ & $0.0208(8)$ & $0.0148(8)$ & $0.0225(8)$ & $-0.0028(7)$ & $0.0007(6)$ & $0.0005(6)$ \\
\hline $\mathrm{C} 2$ & $0.0212(8)$ & $0.0159(9)$ & $0.0258(8)$ & $0.0046(7)$ & $-0.0001(7)$ & $0.0011(7)$ \\
\hline $\mathrm{C} 3$ & $0.0231(9)$ & $0.0283(11)$ & $0.0409(11)$ & $0.0037(8)$ & $-0.0039(8)$ & $0.0035(9)$ \\
\hline $\mathrm{C} 4$ & $0.0265(8)$ & $0.0148(9)$ & $0.0191(8)$ & $0.0010(7)$ & $0.0022(6)$ & $-0.0021(6)$ \\
\hline $\mathrm{C} 5$ & $0.0516(12)$ & $0.0236(10)$ & $0.0216(9)$ & $-0.0026(9)$ & $0.0099(8)$ & $-0.0019(8)$ \\
\hline C6 & $0.0255(9)$ & $0.0194(10)$ & $0.0348(10)$ & $-0.0016(7)$ & $0.0083(8)$ & $0.0002(8)$ \\
\hline $\mathrm{C} 7$ & $0.0277(9)$ & $0.0250(10)$ & $0.0241(9)$ & $0.0055(8)$ & $0.0061(7)$ & $0.0005(7)$ \\
\hline $\mathrm{C} 10$ & $0.0209(8)$ & $0.0165(8)$ & $0.0194(7)$ & $-0.0024(7)$ & $0.0012(6)$ & $-0.0009(6)$ \\
\hline C11 & $0.0194(8)$ & $0.0227(10)$ & $0.0225(8)$ & $-0.0037(7)$ & $0.0024(6)$ & $-0.0004(7)$ \\
\hline $\mathrm{C} 12$ & $0.0194(8)$ & $0.0235(9)$ & $0.0182(8)$ & $0.0007(7)$ & $0.0011(6)$ & $-0.0046(7)$ \\
\hline $\mathrm{C} 13$ & $0.0211(8)$ & $0.0341(11)$ & $0.0216(8)$ & $0.0050(8)$ & $0.0013(7)$ & $-0.0061(7)$ \\
\hline C14 & $0.0283(9)$ & $0.0345(11)$ & $0.0216(8)$ & $0.0148(8)$ & $-0.0018(7)$ & $-0.0042(8)$ \\
\hline C15 & $0.0390(10)$ & $0.0253(10)$ & $0.0201(8)$ & $0.0117(8)$ & $0.0026(7)$ & $0.0031(7)$ \\
\hline C16 & $0.0275(9)$ & $0.0256(10)$ & $0.0189(8)$ & $0.0042(7)$ & $0.0048(7)$ & $0.0027(7)$ \\
\hline $\mathrm{C} 17$ & $0.0206(8)$ & $0.0206(9)$ & $0.0152(7)$ & $0.0024(7)$ & $0.0011(6)$ & $-0.0019(6)$ \\
\hline $\mathrm{C} 1 \mathrm{~A}$ & $0.0253(8)$ & $0.0244(9)$ & $0.0166(8)$ & $0.0014(7)$ & $0.0053(6)$ & $0.0000(7)$ \\
\hline $\mathrm{C} 2 \mathrm{~A}$ & $0.0268(8)$ & $0.0181(9)$ & $0.0197(8)$ & $-0.0009(7)$ & $0.0006(7)$ & $-0.0026(7)$ \\
\hline $\mathrm{C} 3 \mathrm{~A}$ & $0.0372(10)$ & $0.0223(10)$ & $0.0301(9)$ & $-0.0035(8)$ & $0.0034(8)$ & $0.0017(8)$ \\
\hline $\mathrm{C} 4 \mathrm{~A}$ & $0.0296(9)$ & $0.0271(10)$ & $0.0177(8)$ & $-0.0012(8)$ & $-0.0064(7)$ & $0.0010(7)$ \\
\hline $\mathrm{C} 5 \mathrm{~A}$ & $0.0275(9)$ & $0.0354(12)$ & $0.0338(10)$ & $0.0040(8)$ & $-0.0064(8)$ & $0.0004(9)$ \\
\hline C6A & $0.0291(9)$ & $0.0246(10)$ & $0.0237(9)$ & $-0.0051(8)$ & $-0.0004(7)$ & $-0.0022(8)$ \\
\hline C7A & $0.0211(8)$ & $0.0295(10)$ & $0.0198(8)$ & $-0.0006(7)$ & $0.0034(6)$ & $-0.0037(7)$ \\
\hline $\mathrm{C} 10 \mathrm{~A}$ & $0.0239(8)$ & $0.0183(8)$ & $0.0177(8)$ & $-0.0005(7)$ & $0.0063(6)$ & $-0.0015(6)$ \\
\hline C11A & $0.0225(8)$ & $0.0211(9)$ & $0.0244(8)$ & $-0.0027(7)$ & $0.0097(7)$ & $-0.0041(7)$ \\
\hline C12A & $0.0209(8)$ & $0.0158(9)$ & $0.0242(8)$ & $-0.0003(6)$ & $0.0050(7)$ & $-0.0067(6)$ \\
\hline C13A & $0.0182(8)$ & $0.0244(10)$ & $0.0358(10)$ & $0.0015(7)$ & $0.0040(7)$ & $-0.0085(8)$ \\
\hline C14A & $0.0227(9)$ & $0.0277(10)$ & $0.0335(10)$ & $0.0079(7)$ & $-0.0063(7)$ & $-0.0081(8)$ \\
\hline $\mathrm{C} 15 \mathrm{~A}$ & $0.0307(9)$ & $0.0213(10)$ & $0.0239(8)$ & $0.0056(8)$ & $-0.0031(7)$ & $-0.0024(7)$ \\
\hline C16A & $0.0226(8)$ & $0.0172(9)$ & $0.0218(8)$ & $0.0021(7)$ & $0.0028(6)$ & $-0.0024(6)$ \\
\hline C17A & $0.0188(8)$ & $0.0148(8)$ & $0.0203(8)$ & $0.0008(6)$ & $0.0021(6)$ & $-0.0047(7)$ \\
\hline
\end{tabular}

Geometric parameters $\left(\AA,{ }^{\circ}\right)$

\begin{tabular}{llll}
\hline Al1-N1 & $1.8879(14)$ & $\mathrm{C} 12-\mathrm{C} 17$ & $1.422(2)$ \\
A11-C6 & $1.957(2)$ & $\mathrm{C} 13-\mathrm{C} 14$ & $1.375(3)$ \\
A11-C7 & $1.9686(19)$ & $\mathrm{C} 13-\mathrm{H} 13$ & 0.9500 \\
A11-N2 & $2.0355(15)$ & $\mathrm{C} 14-\mathrm{C} 15$ & $1.403(3)$ \\
A11A-N1A & $1.8779(15)$ & $\mathrm{C} 14-\mathrm{H} 14$ & 0.9500 \\
A11A-C6A & $1.960(2)$ & $\mathrm{C} 15-\mathrm{C} 16$ & $1.386(3)$ \\
A11A-C7A & $1.9610(18)$ & $\mathrm{C} 15-\mathrm{H} 15$ & 0.9500 \\
A11A-N2A & $2.0397(16)$ & $\mathrm{C} 16-\mathrm{C} 17$ & $1.391(3)$ \\
N1-C10 & $1.382(2)$ & $\mathrm{C} 16-\mathrm{H} 16$ & 0.9500 \\
N1-C17 & $1.384(2)$ & $\mathrm{C} 1 \mathrm{~A}-\mathrm{C} 10 \mathrm{~A}$ & $1.501(2)$
\end{tabular}




\begin{tabular}{|c|c|c|c|}
\hline $\mathrm{N} 1 \mathrm{~A}-\mathrm{C} 17 \mathrm{~A}$ & $1.378(2)$ & $\mathrm{C} 1 \mathrm{~A}-\mathrm{H} 1 \mathrm{D}$ & 0.9900 \\
\hline $\mathrm{N} 1 \mathrm{~A}-\mathrm{C} 10 \mathrm{~A}$ & $1.384(2)$ & $\mathrm{C} 1 \mathrm{~A}-\mathrm{H} 1 \mathrm{E}$ & 0.9900 \\
\hline $\mathrm{N} 2-\mathrm{C} 2$ & $1.499(2)$ & $\mathrm{C} 2 \mathrm{~A}-\mathrm{C} 3 \mathrm{~A}$ & $1.521(3)$ \\
\hline $\mathrm{N} 2-\mathrm{C} 1$ & $1.504(2)$ & $\mathrm{C} 2 \mathrm{~A}-\mathrm{H} 2 \mathrm{D}$ & 0.9900 \\
\hline $\mathrm{N} 2-\mathrm{C} 4$ & $1.510(2)$ & $\mathrm{C} 2 \mathrm{~A}-\mathrm{H} 2 \mathrm{E}$ & 0.9900 \\
\hline $\mathrm{N} 2 \mathrm{~A}-\mathrm{C} 4 \mathrm{~A}$ & $1.501(2)$ & $\mathrm{C} 3 \mathrm{~A}-\mathrm{H} 3 \mathrm{D}$ & 0.9800 \\
\hline $\mathrm{N} 2 \mathrm{~A}-\mathrm{C} 2 \mathrm{~A}$ & $1.509(2)$ & $\mathrm{C} 3 \mathrm{~A}-\mathrm{H} 3 \mathrm{E}$ & 0.9800 \\
\hline $\mathrm{N} 2 \mathrm{~A}-\mathrm{C} 1 \mathrm{~A}$ & $1.509(2)$ & $\mathrm{C} 3 \mathrm{~A}-\mathrm{H} 3 \mathrm{~F}$ & 0.9800 \\
\hline $\mathrm{C} 1-\mathrm{C} 10$ & $1.500(2)$ & $\mathrm{C} 4 \mathrm{~A}-\mathrm{C} 5 \mathrm{~A}$ & $1.511(3)$ \\
\hline $\mathrm{C} 1-\mathrm{H} 1 \mathrm{~A}$ & 0.9900 & $\mathrm{C} 4 \mathrm{~A}-\mathrm{H} 4 \mathrm{D}$ & 0.9900 \\
\hline $\mathrm{C} 1-\mathrm{H} 1 \mathrm{~B}$ & 0.9900 & $\mathrm{C} 4 \mathrm{~A}-\mathrm{H} 4 \mathrm{E}$ & 0.9900 \\
\hline $\mathrm{C} 2-\mathrm{C} 3$ & $1.515(3)$ & $\mathrm{C} 5 \mathrm{~A}-\mathrm{H} 5 \mathrm{D}$ & 0.9800 \\
\hline $\mathrm{C} 2-\mathrm{H} 2 \mathrm{~A}$ & 0.9900 & $\mathrm{C} 5 \mathrm{~A}-\mathrm{H} 5 \mathrm{E}$ & 0.9800 \\
\hline $\mathrm{C} 2-\mathrm{H} 2 \mathrm{~B}$ & 0.9900 & $\mathrm{C} 5 \mathrm{~A}-\mathrm{H} 5 \mathrm{~F}$ & 0.9800 \\
\hline $\mathrm{C} 3-\mathrm{H} 3 \mathrm{~A}$ & 0.9800 & C6A-H6D & 0.9800 \\
\hline $\mathrm{C} 3-\mathrm{H} 3 \mathrm{~B}$ & 0.9800 & C6A-H6E & 0.9800 \\
\hline $\mathrm{C} 3-\mathrm{H} 3 \mathrm{C}$ & 0.9800 & $\mathrm{C} 6 \mathrm{~A}-\mathrm{H} 6 \mathrm{~F}$ & 0.9800 \\
\hline $\mathrm{C} 4-\mathrm{C} 5$ & $1.520(2)$ & C7A-H7D & 0.9800 \\
\hline $\mathrm{C} 4-\mathrm{H} 4 \mathrm{~A}$ & 0.9900 & $\mathrm{C} 7 \mathrm{~A}-\mathrm{H} 7 \mathrm{E}$ & 0.9800 \\
\hline $\mathrm{C} 4-\mathrm{H} 4 \mathrm{~B}$ & 0.9900 & $\mathrm{C} 7 \mathrm{~A}-\mathrm{H} 7 \mathrm{~F}$ & 0.9800 \\
\hline $\mathrm{C} 5-\mathrm{H} 5 \mathrm{~A}$ & 0.9800 & $\mathrm{C} 10 \mathrm{~A}-\mathrm{C} 11 \mathrm{~A}$ & $1.366(2)$ \\
\hline $\mathrm{C} 5-\mathrm{H} 5 \mathrm{~B}$ & 0.9800 & $\mathrm{C} 11 \mathrm{~A}-\mathrm{C} 12 \mathrm{~A}$ & $1.433(3)$ \\
\hline $\mathrm{C} 5-\mathrm{H} 5 \mathrm{C}$ & 0.9800 & $\mathrm{C} 11 \mathrm{~A}-\mathrm{H} 11 \mathrm{~A}$ & 0.9500 \\
\hline $\mathrm{C} 6-\mathrm{H} 6 \mathrm{~A}$ & 0.9800 & $\mathrm{C} 12 \mathrm{~A}-\mathrm{C} 13 \mathrm{~A}$ & $1.404(2)$ \\
\hline C6-H6B & 0.9800 & $\mathrm{C} 12 \mathrm{~A}-\mathrm{C} 17 \mathrm{~A}$ & $1.428(2)$ \\
\hline $\mathrm{C} 6-\mathrm{H} 6 \mathrm{C}$ & 0.9800 & $\mathrm{C} 13 \mathrm{~A}-\mathrm{C} 14 \mathrm{~A}$ & $1.382(3)$ \\
\hline $\mathrm{C} 7-\mathrm{H} 7 \mathrm{~A}$ & 0.9800 & $\mathrm{C} 13 \mathrm{~A}-\mathrm{H} 13 \mathrm{~A}$ & 0.9500 \\
\hline $\mathrm{C} 7-\mathrm{H} 7 \mathrm{~B}$ & 0.9800 & $\mathrm{C} 14 \mathrm{~A}-\mathrm{C} 15 \mathrm{~A}$ & $1.409(3)$ \\
\hline $\mathrm{C} 7-\mathrm{H} 7 \mathrm{C}$ & 0.9800 & $\mathrm{C} 14 \mathrm{~A}-\mathrm{H} 14 \mathrm{~A}$ & 0.9500 \\
\hline $\mathrm{C} 10-\mathrm{C} 11$ & $1.365(2)$ & $\mathrm{C} 15 \mathrm{~A}-\mathrm{C} 16 \mathrm{~A}$ & $1.380(2)$ \\
\hline $\mathrm{C} 11-\mathrm{C} 12$ & $1.433(3)$ & $\mathrm{C} 15 \mathrm{~A}-\mathrm{H} 15 \mathrm{~A}$ & 0.9500 \\
\hline C11-H11 & 0.9500 & $\mathrm{C} 16 \mathrm{~A}-\mathrm{C} 17 \mathrm{~A}$ & $1.398(2)$ \\
\hline $\mathrm{C} 12-\mathrm{C} 13$ & $1.404(2)$ & $\mathrm{C} 16 \mathrm{~A}-\mathrm{H} 16 \mathrm{~A}$ & 0.9500 \\
\hline $\mathrm{N} 1-\mathrm{A} 11-\mathrm{C} 6$ & $111.91(8)$ & $\mathrm{C} 14-\mathrm{C} 13-\mathrm{C} 12$ & $119.17(18)$ \\
\hline $\mathrm{N} 1-\mathrm{A} 11-\mathrm{C} 7$ & $116.33(8)$ & $\mathrm{C} 14-\mathrm{C} 13-\mathrm{H} 13$ & 120.4 \\
\hline C6-A11-C7 & $117.73(8)$ & $\mathrm{C} 12-\mathrm{C} 13-\mathrm{H} 13$ & 120.4 \\
\hline $\mathrm{N} 1-\mathrm{A} 11-\mathrm{N} 2$ & $85.25(6)$ & $\mathrm{C} 13-\mathrm{C} 14-\mathrm{C} 15$ & $121.16(17)$ \\
\hline C6-A11-N2 & $115.96(7)$ & C13-C14-H14 & 119.4 \\
\hline $\mathrm{C} 7-\mathrm{A} 11-\mathrm{N} 2$ & $105.14(7)$ & $\mathrm{C} 15-\mathrm{C} 14-\mathrm{H} 14$ & 119.4 \\
\hline $\mathrm{N} 1 \mathrm{~A}-\mathrm{A} 11 \mathrm{~A}-\mathrm{C} 6 \mathrm{~A}$ & $113.03(8)$ & $\mathrm{C} 16-\mathrm{C} 15-\mathrm{C} 14$ & $120.99(19)$ \\
\hline $\mathrm{N} 1 \mathrm{~A}-\mathrm{A} 11 \mathrm{~A}-\mathrm{C} 7 \mathrm{~A}$ & $114.12(7)$ & $\mathrm{C} 16-\mathrm{C} 15-\mathrm{H} 15$ & 119.5 \\
\hline $\mathrm{C} 6 \mathrm{~A}-\mathrm{A} 11 \mathrm{~A}-\mathrm{C} 7 \mathrm{~A}$ & $118.00(8)$ & C14-C15-H15 & 119.5 \\
\hline $\mathrm{N} 1 \mathrm{~A}-\mathrm{A} 11 \mathrm{~A}-\mathrm{N} 2 \mathrm{~A}$ & $85.91(6)$ & $\mathrm{C} 15-\mathrm{C} 16-\mathrm{C} 17$ & $118.25(17)$ \\
\hline $\mathrm{C} 6 \mathrm{~A}-\mathrm{A} 11 \mathrm{~A}-\mathrm{N} 2 \mathrm{~A}$ & $108.30(7)$ & $\mathrm{C} 15-\mathrm{C} 16-\mathrm{H} 16$ & 120.9 \\
\hline $\mathrm{C} 7 \mathrm{~A}-\mathrm{A} 11 \mathrm{~A}-\mathrm{N} 2 \mathrm{~A}$ & $112.91(8)$ & $\mathrm{C} 17-\mathrm{C} 16-\mathrm{H} 16$ & 120.9 \\
\hline $\mathrm{C} 10-\mathrm{N} 1-\mathrm{C} 17$ & $105.83(13)$ & $\mathrm{N} 1-\mathrm{C} 17-\mathrm{C} 16$ & $129.35(16)$ \\
\hline $\mathrm{C} 10-\mathrm{N} 1-\mathrm{A} 11$ & $112.84(11)$ & $\mathrm{N} 1-\mathrm{C} 17-\mathrm{C} 12$ & $109.32(16)$ \\
\hline
\end{tabular}




$\begin{array}{ll}\text { C17-N1-A11 } & 139.57(13) \\ \text { C17A-N1A-C10A } & 106.15(14) \\ \text { C17A-N1A-A11A } & 140.50(12) \\ \text { C10A-N1A-A11A } & 113.18(11) \\ \text { C2-N2-C1 } & 108.22(13) \\ \text { C2-N2-C4 } & 112.02(12) \\ \text { C1-N2-C4 } & 110.43(13) \\ \text { C2-N2-A11 } & 115.63(10) \\ \text { C1-N2-A11 } & 101.69(10) \\ \text { C4-N2-A11 } & 108.35(10) \\ \text { C4A-N2A-C2A } & 112.02(14) \\ \text { C4A-N2A-C1A } & 109.27(13) \\ \text { C2A-N2A-C1A } & 110.02(13) \\ \text { C4A-N2A-A11A } & 114.30(11) \\ \text { C2A-N2A-A11A } & 108.47(10) \\ \text { C1A-N2A-A11A } & 102.31(11) \\ \text { C10-C1-N2 } & 107.43(14) \\ \text { C10-C1-H1A } & 110.2 \\ \text { N2-C1-H1A } & 110.2 \\ \text { C10-C1-H1B } & 110.2 \\ \text { N2-C1-H1B } & 110.2 \\ \text { H1A-C1-H1B } & 108.5 \\ \text { N2-C2-C3 } & 113.28(15) \\ \text { N2-C2-H2A } & 108.9 \\ \text { C3-C2-H2A } & 108.9 \\ \text { N2-C2-H2B } & 108.9 \\ \text { C3-C2-H2B } & 108.9 \\ \text { H2A-C2-H2B } & 107.7 \\ \text { C2-C3-H3A } & 109.5 \\ \text { C2-C3-H3B } & 109.5 \\ \text { H3A-C3-H3B } & 109.5 \\ \text { C2-C3-H3C } & 109.5 \\ \text { H3A-C3-H3C } & 109.5 \\ \text { H3B-C3-H3C } & 109.5 \\ \text { N2-C4-C5 } & 115.68(15) \\ \text { N2-C4-H4A } & 108.4 \\ \text { C5-C4-H4A } & 108.4 \\ \text { N2-C4-H4B } & 108.4 \\ \text { C5-C4-H4B } & 108.4 \\ \text { H4A-C4-H4B } & 107.4 \\ \text { C4-C5-H5A } & 109.5 \\ \text { C11-C6-H6A } 4-C 5-H 5 B & 109.5 \\ \text { H5A-C5-H5B } & \\ \text { C4-C5-H5C } & 109.5 \\ & \end{array}$

\begin{tabular}{|c|c|}
\hline $\mathrm{C} 16-\mathrm{C} 17-\mathrm{C} 12$ & $121.28(16)$ \\
\hline $\mathrm{C} 10 \mathrm{~A}-\mathrm{C} 1 \mathrm{~A}-\mathrm{N} 2 \mathrm{~A}$ & $108.11(13)$ \\
\hline $\mathrm{C} 10 \mathrm{~A}-\mathrm{C} 1 \mathrm{~A}-\mathrm{H} 1 \mathrm{D}$ & 110.1 \\
\hline $\mathrm{N} 2 \mathrm{~A}-\mathrm{C} 1 \mathrm{~A}-\mathrm{H} 1 \mathrm{D}$ & 110.1 \\
\hline $\mathrm{C} 10 \mathrm{~A}-\mathrm{C} 1 \mathrm{~A}-\mathrm{H} 1 \mathrm{E}$ & 110.1 \\
\hline $\mathrm{N} 2 \mathrm{~A}-\mathrm{C} 1 \mathrm{~A}-\mathrm{H} 1 \mathrm{E}$ & 110.1 \\
\hline $\mathrm{H} 1 \mathrm{D}-\mathrm{C} 1 \mathrm{~A}-\mathrm{H} 1 \mathrm{E}$ & 108.4 \\
\hline $\mathrm{N} 2 \mathrm{~A}-\mathrm{C} 2 \mathrm{~A}-\mathrm{C} 3 \mathrm{~A}$ & $115.85(14)$ \\
\hline $\mathrm{N} 2 \mathrm{~A}-\mathrm{C} 2 \mathrm{~A}-\mathrm{H} 2 \mathrm{D}$ & 108.3 \\
\hline $\mathrm{C} 3 \mathrm{~A}-\mathrm{C} 2 \mathrm{~A}-\mathrm{H} 2 \mathrm{D}$ & 108.3 \\
\hline $\mathrm{N} 2 \mathrm{~A}-\mathrm{C} 2 \mathrm{~A}-\mathrm{H} 2 \mathrm{E}$ & 108.3 \\
\hline $\mathrm{C} 3 \mathrm{~A}-\mathrm{C} 2 \mathrm{~A}-\mathrm{H} 2 \mathrm{E}$ & 108.3 \\
\hline $\mathrm{H} 2 \mathrm{D}-\mathrm{C} 2 \mathrm{~A}-\mathrm{H} 2 \mathrm{E}$ & 107.4 \\
\hline $\mathrm{C} 2 \mathrm{~A}-\mathrm{C} 3 \mathrm{~A}-\mathrm{H} 3 \mathrm{D}$ & 109.5 \\
\hline $\mathrm{C} 2 \mathrm{~A}-\mathrm{C} 3 \mathrm{~A}-\mathrm{H} 3 \mathrm{E}$ & 109.5 \\
\hline $\mathrm{H} 3 \mathrm{D}-\mathrm{C} 3 \mathrm{~A}-\mathrm{H} 3 \mathrm{E}$ & 109.5 \\
\hline $\mathrm{C} 2 \mathrm{~A}-\mathrm{C} 3 \mathrm{~A}-\mathrm{H} 3 \mathrm{~F}$ & 109.5 \\
\hline $\mathrm{H} 3 \mathrm{D}-\mathrm{C} 3 \mathrm{~A}-\mathrm{H} 3 \mathrm{~F}$ & 109.5 \\
\hline $\mathrm{H} 3 \mathrm{E}-\mathrm{C} 3 \mathrm{~A}-\mathrm{H} 3 \mathrm{~F}$ & 109.5 \\
\hline $\mathrm{N} 2 \mathrm{~A}-\mathrm{C} 4 \mathrm{~A}-\mathrm{C} 5 \mathrm{~A}$ & $113.37(15)$ \\
\hline $\mathrm{N} 2 \mathrm{~A}-\mathrm{C} 4 \mathrm{~A}-\mathrm{H} 4 \mathrm{D}$ & 108.9 \\
\hline $\mathrm{C} 5 \mathrm{~A}-\mathrm{C} 4 \mathrm{~A}-\mathrm{H} 4 \mathrm{D}$ & 108.9 \\
\hline $\mathrm{N} 2 \mathrm{~A}-\mathrm{C} 4 \mathrm{~A}-\mathrm{H} 4 \mathrm{E}$ & 108.9 \\
\hline $\mathrm{C} 5 \mathrm{~A}-\mathrm{C} 4 \mathrm{~A}-\mathrm{H} 4 \mathrm{E}$ & 108.9 \\
\hline $\mathrm{H} 4 \mathrm{D}-\mathrm{C} 4 \mathrm{~A}-\mathrm{H} 4 \mathrm{E}$ & 107.7 \\
\hline $\mathrm{C} 4 \mathrm{~A}-\mathrm{C} 5 \mathrm{~A}-\mathrm{H} 5 \mathrm{D}$ & 109.5 \\
\hline $\mathrm{C} 4 \mathrm{~A}-\mathrm{C} 5 \mathrm{~A}-\mathrm{H} 5 \mathrm{E}$ & 109.5 \\
\hline $\mathrm{H} 5 \mathrm{D}-\mathrm{C} 5 \mathrm{~A}-\mathrm{H} 5 \mathrm{E}$ & 109.5 \\
\hline $\mathrm{C} 4 \mathrm{~A}-\mathrm{C} 5 \mathrm{~A}-\mathrm{H} 5 \mathrm{~F}$ & 109.5 \\
\hline $\mathrm{H} 5 \mathrm{D}-\mathrm{C} 5 \mathrm{~A}-\mathrm{H} 5 \mathrm{~F}$ & 109.5 \\
\hline $\mathrm{H} 5 \mathrm{E}-\mathrm{C} 5 \mathrm{~A}-\mathrm{H} 5 \mathrm{~F}$ & 109.5 \\
\hline $\mathrm{A} 11 \mathrm{~A}-\mathrm{C} 6 \mathrm{~A}-\mathrm{H} 6 \mathrm{D}$ & 109.5 \\
\hline $\mathrm{A} 11 \mathrm{~A}-\mathrm{C} 6 \mathrm{~A}-\mathrm{H} 6 \mathrm{E}$ & 109.5 \\
\hline $\mathrm{H} 6 \mathrm{D}-\mathrm{C} 6 \mathrm{~A}-\mathrm{H} 6 \mathrm{E}$ & 109.5 \\
\hline $\mathrm{A} 11 \mathrm{~A}-\mathrm{C} 6 \mathrm{~A}-\mathrm{H} 6 \mathrm{~F}$ & 109.5 \\
\hline $\mathrm{H} 6 \mathrm{D}-\mathrm{C} 6 \mathrm{~A}-\mathrm{H} 6 \mathrm{~F}$ & 109.5 \\
\hline $\mathrm{H} 6 \mathrm{E}-\mathrm{C} 6 \mathrm{~A}-\mathrm{H} 6 \mathrm{~F}$ & 109.5 \\
\hline $\mathrm{A} 11 \mathrm{~A}-\mathrm{C} 7 \mathrm{~A}-\mathrm{H} 7 \mathrm{D}$ & 109.5 \\
\hline $\mathrm{Al1A}-\mathrm{C} 7 \mathrm{~A}-\mathrm{H} 7 \mathrm{E}$ & 109.5 \\
\hline $\mathrm{H} 7 \mathrm{D}-\mathrm{C} 7 \mathrm{~A}-\mathrm{H} 7 \mathrm{E}$ & 109.5 \\
\hline $\mathrm{A} 11 \mathrm{~A}-\mathrm{C} 7 \mathrm{~A}-\mathrm{H} 7 \mathrm{~F}$ & 109.5 \\
\hline $\mathrm{H} 7 \mathrm{D}-\mathrm{C} 7 \mathrm{~A}-\mathrm{H} 7 \mathrm{~F}$ & 109.5 \\
\hline $\mathrm{H} 7 \mathrm{E}-\mathrm{C} 7 \mathrm{~A}-\mathrm{H} 7 \mathrm{~F}$ & 109.5 \\
\hline $\mathrm{C} 11 \mathrm{~A}-\mathrm{C} 10 \mathrm{~A}-\mathrm{N} 1 \mathrm{~A}$ & $112.32(15)$ \\
\hline $\mathrm{C} 11 \mathrm{~A}-\mathrm{C} 10 \mathrm{~A}-\mathrm{C} 1 \mathrm{~A}$ & $132.80(16)$ \\
\hline $\mathrm{N} 1 \mathrm{~A}-\mathrm{C} 10 \mathrm{~A}-\mathrm{C} 1 \mathrm{~A}$ & $114.84(14)$ \\
\hline $\mathrm{C} 10 \mathrm{~A}-\mathrm{C} 11 \mathrm{~A}-\mathrm{C} 12 \mathrm{~A}$ & $106.03(15)$ \\
\hline $\mathrm{C} 10 \mathrm{~A}-\mathrm{C} 11 \mathrm{~A}-\mathrm{H} 11 \mathrm{~A}$ & 127.0 \\
\hline
\end{tabular}




\section{supporting information}

$\begin{array}{llll}\text { H6A-C6-H6B } & 109.5 & \mathrm{C} 12 \mathrm{~A}-\mathrm{C} 11 \mathrm{~A}-\mathrm{H} 11 \mathrm{~A} & 127.0 \\ \mathrm{~A} 11-\mathrm{C} 6-\mathrm{H} 6 \mathrm{C} & 109.5 & \mathrm{C} 13 \mathrm{~A}-\mathrm{C} 12 \mathrm{~A}-\mathrm{C} 17 \mathrm{~A} & 118.82(17) \\ \mathrm{H} 6 \mathrm{~A}-\mathrm{C} 6-\mathrm{H} 6 \mathrm{C} & 109.5 & \mathrm{C} 13 \mathrm{~A}-\mathrm{C} 12 \mathrm{~A}-\mathrm{C} 11 \mathrm{~A} & 135.04(17) \\ \mathrm{H} 6 \mathrm{~B}-\mathrm{C} 6-\mathrm{H} 6 \mathrm{C} & 109.5 & \mathrm{C} 17 \mathrm{~A}-\mathrm{C} 12 \mathrm{~A}-\mathrm{C} 11 \mathrm{~A} & 106.14(15) \\ \mathrm{A} 11-\mathrm{C} 7-\mathrm{H} 7 \mathrm{~A} & 109.5 & \mathrm{C} 14 \mathrm{~A}-\mathrm{C} 13 \mathrm{~A}-\mathrm{C} 12 \mathrm{~A} & 119.22(17) \\ \text { A11-C7-H7B } & 109.5 & \mathrm{C} 14 \mathrm{~A}-\mathrm{C} 13 \mathrm{~A}-\mathrm{H} 13 \mathrm{~A} & 120.4 \\ \mathrm{H} 7 \mathrm{~A}-\mathrm{C} 7-\mathrm{H} 7 \mathrm{~B} & 109.5 & \mathrm{C} 12 \mathrm{~A}-\mathrm{C} 13 \mathrm{~A}-\mathrm{H} 13 \mathrm{~A} & 120.4 \\ \mathrm{~A} 11-\mathrm{C} 7-\mathrm{H} 7 \mathrm{C} & 109.5 & \mathrm{C} 13 \mathrm{~A}-\mathrm{C} 14 \mathrm{~A}-\mathrm{C} 15 \mathrm{~A} & 121.12(17) \\ \mathrm{H} 7 \mathrm{~A}-\mathrm{C} 7-\mathrm{H} 7 \mathrm{C} & 109.5 & \mathrm{C} 13 \mathrm{~A}-\mathrm{C} 14 \mathrm{~A}-\mathrm{H} 14 \mathrm{~A} & 119.4 \\ \mathrm{H} 7 \mathrm{~B}-\mathrm{C} 7-\mathrm{H} 7 \mathrm{C} & 109.5 & \mathrm{C} 15 \mathrm{~A}-\mathrm{C} 14 \mathrm{~A}-\mathrm{H} 14 \mathrm{~A} & 119.4 \\ \mathrm{C} 11-\mathrm{C} 10-\mathrm{N} 1 & \mathrm{C} 16 \mathrm{~A}-\mathrm{C} 15 \mathrm{~A}-\mathrm{C} 14 \mathrm{~A} & 121.18(18) \\ \mathrm{C} 11-\mathrm{C} 10-\mathrm{C} 1 & 112.64(15) & \mathrm{C} 16 \mathrm{~A}-\mathrm{C} 15 \mathrm{~A}-\mathrm{H} 15 \mathrm{~A} & 119.4 \\ \mathrm{~N} 1-\mathrm{C} 10-\mathrm{C} 1 & 132.87(16) & \mathrm{C} 14 \mathrm{~A}-\mathrm{C} 15 \mathrm{~A}-\mathrm{H} 15 \mathrm{~A} & 119.4 \\ \mathrm{C} 10-\mathrm{C} 11-\mathrm{C} 16 \mathrm{~A}-\mathrm{C} 17 \mathrm{~A} & 118.04(17) \\ \mathrm{C} 10-\mathrm{C} 11-\mathrm{H} 11 & 114.36(14) & \mathrm{C} 15 \mathrm{~A}-\mathrm{C} 16 \mathrm{~A}-\mathrm{H} 16 \mathrm{~A} & 121.0 \\ \mathrm{C} 12-\mathrm{C} 11-\mathrm{H} 11 & 105.78(15) & \mathrm{C} 17 \mathrm{~A}-\mathrm{C} 16 \mathrm{~A}-\mathrm{H} 16 \mathrm{~A} & 121.0 \\ \mathrm{C} 13-\mathrm{C} 12-\mathrm{C} 17 & 127.1 & \mathrm{~N} 1 \mathrm{~A}-\mathrm{C} 17 \mathrm{~A}-\mathrm{C} 16 \mathrm{~A} & 129.05(16) \\ \mathrm{C} 13-\mathrm{C} 12-\mathrm{C} 11 & 127.1 & \mathrm{~N} 1 \mathrm{~A}-\mathrm{C} 17 \mathrm{~A}-\mathrm{C} 12 \mathrm{~A} & 109.34(15) \\ \mathrm{C} 17-\mathrm{C} 12-\mathrm{C} 11 & 119.11(18) & \mathrm{C} 16 \mathrm{~A}-\mathrm{C} 17 \mathrm{~A}-\mathrm{C} 12 \mathrm{~A} & 121.61(16)\end{array}$

Hydrogen-bond geometry $\left(A,{ }^{o}\right)$

$\mathrm{Cg} 1$ is the centroid of the $\mathrm{C} 12 A-\mathrm{C} 17 A$ ring.

\begin{tabular}{lllll}
\hline$D-\mathrm{H} \cdots A$ & $D-\mathrm{H}$ & $\mathrm{H} \cdots A$ & $D \cdots A$ & $D-\mathrm{H} \cdots A$ \\
\hline $\mathrm{C} 5-\mathrm{H} 5 A \cdots C g 1$ & 0.98 & 2.57 & $3.470(2)$ & 153 \\
$\mathrm{C} 2-\mathrm{H} 2 B \cdots C g 1^{\mathrm{i}}$ & 0.99 & 2.55 & $3.434(2)$ & 149 \\
\hline
\end{tabular}

Symmetry code: (i) $-x+1, y-1 / 2,-z+1$. 